Proyecciones

Vol. $18, N^{\circ} 2$, pp. 175-182, December 1999

Universidad Católica del Norte

Antofagasta - Chile

\title{
ON CHARACTERIZATION OF REFLECTION AND COREFLECTION IN CATEGORIES
}

\author{
HAMZA A. S. ABUJABAL \\ King Abdul Aziz University, Saudi Arabia \\ and \\ S. M. A. ZAIDI \\ Aligrah Muslim University, India
}

\begin{abstract}
The main purpose of the present paper is to present a criteria of reflection and its dual through generalized pushout (GPO) and generalized pullback (GPB) structures. Several results related to reflective and coreflective subcategories are obtained.
\end{abstract}

KEY WORDS AND PHRASES : Reflection, Reflective subcategory, dense subcategory, GPB category, GPO category, GPB functor, GPO functor, generator.

1991 AMS Subject classification : 18D15. 


\section{Introduction}

The concepts of reflection and reflective subcategory and their duals introduced by P.J. Freyd (see [1]). Here we have obtained the existence of reflection and coreflection by generalized pushout $(G P O)$ and generalized pullback $(G P B)$ respectively. We have also discussed several other properties of reflective and coreflective subcategories.

\section{Preliminares}

In this paper, we will clarify only few preliminary notions frequently used in the development of our results. For other concepts we refer [2], [3] and [4].

Definition 1 : A non-empty family of morphisms $\left\{\alpha_{i}: A \rightarrow B_{i}\right\}_{I}$ in a category $\mathcal{C}$ is called a left cone over $I$ with vertex $A$ and base objects $B_{i}, i \in I$. We will denote it by $\left[A, \alpha_{i}, B_{i}\right]_{I}$.

Dually, we can define a right cone.

Definition 2 : In a category $C$, a left cone $\left[P, \alpha_{i}, B_{i}\right]_{I}$ is called the generalized pullback $(G P B)$ of the right cone $\left[B_{i}, \beta_{i}, A\right]_{I}$ if and only if

$G P B_{1}: \beta_{j \alpha j}=\beta_{k \alpha k}$ for all $j, k \in I$,

$G P B_{2}:$ For any other left cone $\left[X, f_{i}, B_{i}\right]_{I}$ in $\mathcal{C}$ with $\beta_{j} f_{j}=\beta_{k} f_{k}$ for all $j, k \in I$, there exists a unique morphism $\eta: X \rightarrow P$ in $\mathcal{C}$ such that $\alpha_{i} \eta=f_{i}$ for all $i \in I$.

Dually, we can define generalized pushout $(G P O)$.

Definition 3 : Let $C$ be a category and $\mathcal{A}$ a subcategory of $\mathcal{C}$. For an object $C \in \mathcal{C}$, an object $\bar{C} \in \mathcal{A}$ together with a morphism $\rho_{C}: C \rightarrow \bar{C}$ will be called a reflection of $C$, in $\mathcal{A}$, if for every morphism $\alpha: C \rightarrow A$ with $A \in \mathcal{A}$, there exists a unique morphism $\bar{\alpha}: \bar{C} \rightarrow A$ in $\mathcal{A}$ such that

$$
C \stackrel{\rho C}{\longrightarrow} \bar{C} \stackrel{\bar{\alpha}}{\longrightarrow} A=C \stackrel{\alpha}{\longrightarrow} A .
$$

Dually, an object $\underline{C} \in \mathcal{A}$ together with a morphism $\sigma_{C}: \underline{C} \rightarrow C$ will be called a coreflection of $C$ in $\mathcal{A}$ if for every morphism $\beta: A \rightarrow C$ with $A \in \mathcal{A}$, there exists a unique morphism $\underline{\beta}: A \rightarrow \underline{C}$ in $\mathcal{A}$ such that

$$
A \stackrel{\beta}{\longrightarrow} \underline{C} \stackrel{\sigma C}{\longrightarrow} C=A \stackrel{\alpha}{\longrightarrow} C .
$$




\section{Remark 1 :}

Reflection (coreflection) is unique up to isomorphism.

Definition 4 : If every object of the category $C$ has a reflection (coreflection) in a subcategory $\mathcal{A}$, then $\mathcal{A}$ will be called a reflective (coreflective) subcategory of $\mathcal{C}$.

Definition 5: If $\mathcal{A} \subseteq C$ is a reflective subcategory, then by associating every object of $\mathcal{C}$ to its reflection in $\mathcal{A}$, we can define a functor $\bar{R}: \mathcal{C} \rightarrow$ $\mathcal{A}$.This is called a reflector.

Dually, if $\mathcal{A} \subseteq \mathcal{C}$ is a coreflective subcategory, we can define coreflector $\underline{R}: \mathcal{C} \rightarrow \mathcal{A}$.

Remark 2 : Every reflector is a left adjoint of the inclusion functor and hence a $G P O$ functor.

Dually, every coreflector is a right adjoint of the inclusion functor and hence a $G P B$ functor.

\section{Existence and properties}

Theorem 1 :

Let $\mathcal{A}$ be a subcategory of $C$, and $\left[A, \alpha_{i}, A_{i}\right]_{I}$ be a left cone in $\mathcal{A}$.If its $G P O$ 's exist in $\mathcal{C}$ and $\mathcal{A}$ both, then the vertex of $G P O\left[A, \alpha_{i}, A_{i}\right]_{I}$ in $\mathcal{A}$, is a reflection of the vertex of GPO $\left[A, \alpha_{i}, A_{i}\right]_{I}$ in $\mathcal{C}$.

\section{Proof :}

Suppose,

$$
G P O\left[A, \alpha_{i}, A_{i}\right]_{I}=\left[A_{i}, \beta_{i}, P\right]_{I} \text { in } \mathcal{A},
$$

and

$$
\text { GPO }\left[A, \alpha_{i}, A_{i}\right]_{I}=\left[A_{i}, \gamma_{i}, Q\right]_{I} \text { in } \mathcal{C} .
$$

Since $\mathcal{A} \subseteq \mathcal{C}$, there exists a unique morphism (by Axiom $G P O_{2}$ ) $\eta: Q \rightarrow$ $P \in \mathcal{C}$ such that

$$
\eta \gamma_{i}=\beta_{i}: A_{i} \rightarrow P \text { for all } i \text { in } I .
$$

Now, we show that the object $P \in \mathcal{A}$ together with a morphism $\eta: Q \rightarrow P$ is a reflection of $Q$ in $\mathcal{A}$. For any morphism $f: Q \rightarrow X$ in $\mathcal{C}$ with $X \in \mathcal{A}$, there exists a right cone $\left[A_{i}, f \gamma_{i}, X\right]_{I}$ in $\mathcal{C}$ such that the morphisms

$$
\left(f \gamma_{i}\right) \alpha_{i}=f\left(\gamma_{i \alpha i}\right): A \rightarrow A_{i} \rightarrow X
$$


are the same for all $i \in I$. Further, by Axiom $G P O_{2}$, there exists a unique morphism $\bar{f}: P \rightarrow X$ in $\mathcal{A}$ such that

$$
\bar{f} \beta_{i}=f \gamma_{i}: A_{i} \rightarrow X \text { for all } i \in I,
$$

and so

$$
\bar{f}\left(\eta \gamma_{i}\right)=(\bar{f} \eta) \gamma_{i}=f \gamma_{i} \text { for all } i \in I .
$$

Again, by Axiom $\mathrm{GPO}_{2}$, we get

$$
\bar{f} \eta=f .
$$

Hence, $P$ together with $\eta: Q \rightarrow P$ is a reflection of $Q$.

Dually, we have

Theorem $1^{*}$ : Let $\mathcal{A}$ be a subcategory of $\mathcal{C}$, and $\left[A_{i}, \alpha_{i}, A\right]_{I}$ be a right cone in $\mathcal{A}$, if its $G P B / \mathrm{s}$ exists in $\mathcal{C}$ and $\mathcal{A}$ both. Then the vertex of $G P B\left[A_{i}, \alpha_{i}, A\right]_{I}$ in $\mathcal{A}$ is a coreflection of the vertex of $G P B\left[A_{i}, \alpha_{i}, A\right]_{I}$ in $\mathcal{C}$.

Theorem 2 : Every GPO-dense subcategory of a GPO-category is reflective. Conversely, every reflective subcategory of a GPO-category is also a $G P O$-category.

Proof : Suppose $\mathcal{C}$ is a category and $\mathcal{A}$ is a $G P O$-dense subcategory of $\mathcal{C}$ which is also a $G P O$-category. Let $C$ be any object of $\mathcal{C}$. By dense property, there exists a left cone $\left[A, \alpha_{i}, A_{i}\right]_{I}$ in $\mathcal{A}$ such that $C$ is the vertex of $G P O\left[A, \alpha_{i}, A_{i}\right]_{I}$ in $\mathcal{C}$. Suppose,

$$
G P O\left[A, \alpha_{i}, A_{i}\right]_{I}=\left[A_{i}, \beta_{i}, C\right]_{I} \text { in } \mathcal{C} .
$$

Further, $\mathcal{A}$ is a $G P O$-category, this implies that $G P O\left[A, \alpha_{i}, A_{i}\right]_{I}$ also exists in $A$.Let

$$
G P O\left[A, \alpha_{i}, A_{i}\right]_{I}=\left[A_{i}, \bar{\beta}_{i}, \bar{C}\right]_{I} \text { in } \mathcal{A} \text {. }
$$

By Theorem $1, \bar{C}$ is the reflection of $C$ in $\mathcal{A}$. Hence, $\mathcal{A}$ is a reflective subcategory of $\mathcal{C}$.

Conversely, we assume that $c$ is a $G P O$-category and is a reflective subcategory of $\mathcal{C}$. Consider an arbitrary left cone $\left[A, \alpha_{i}, A_{i}\right]_{I}$ in $\mathcal{A}$ and let

$$
G P O\left[A, \alpha_{i}, A_{i}\right]_{I}=\left[A_{i}, \beta_{i}, P\right]_{I} \text { in } \mathcal{C} .
$$


Let $\bar{P} \in \mathcal{A}$ together with a morphism $\rho_{P}: P \rightarrow \bar{P}$ be a reflection of $P$ in $\mathcal{A}$.Consider the right cone $\left[A_{i}, \rho_{P \beta i}, \bar{P}\right]_{I}$ in $\mathcal{A}$. It is clear that the morphisms

$$
\left(\rho_{P \beta i}\right) \alpha_{i}: A \rightarrow A_{i} \rightarrow \bar{P}
$$

are the same for all $i \in I$ in $\mathcal{A}$. Suppose there is another right cone $\left[A_{i}, f_{i}, X\right]_{I}$ in $\mathcal{A}$ such that the morphisms

$$
f_{i \alpha i}: A \rightarrow A_{i} \rightarrow X
$$

are the same for all $i \in I$ in $\mathcal{A}$. By axiom $G P O_{2}$, there exists a unique morphism $\eta: P \rightarrow X$ in $\mathcal{C}$ such that

$$
\eta \beta_{i}=f_{i}: A_{i} \rightarrow X \text { for all } i \in I .
$$

Further, by definition of reflection, there exists a unique morphism $\bar{\eta}: \bar{P} \rightarrow$ $X$ such that

$$
\begin{gathered}
\bar{\eta} \rho_{p}=\eta \\
\bar{\eta}\left(\rho P \beta_{i}\right)=(\bar{\eta} \rho P) \beta_{i} \\
=\eta \beta_{i}=f_{i} \text { for all } i \in I,
\end{gathered}
$$

and

$$
G P O\left[A, \alpha_{i}, A_{i}\right]_{I}=\left[A_{i}, \rho_{P \beta i}, \bar{P}\right]_{I} \text { in } \mathcal{A} .
$$

Hence, $\mathcal{A}$ is a $G P O$-category.

Dually, we have

Theorem 2* : Every GPO-dense subcategory which is also a $G P B$ - category is coreflective. Conversely, every coreflective subcategory of a $G P B$-category is also a $G P B$-category.

Theorem 3 : Every full reflective subcategory of a $G P B$ - category is also a $G P B$ - category.

Proof : Let $\mathcal{C}$ be a $G P B$-category and $\mathcal{A}$ be its full reflective subcategory. Consider an arbitrary right cone $\left[A_{i}, \alpha_{i}, A_{]}\right.$in $\mathcal{A}$. Suppose

$$
G P B\left[A_{i}, \alpha_{i}, A\right]_{I}=\left[P, \beta_{i}, A_{i}\right]_{I} \text { in } \mathcal{C} \text {. }
$$

Let $\bar{P} \in \mathcal{A}$ together with morphism $\rho_{P}: P \rightarrow \bar{P}$ be a reflection of $P$ in $\mathcal{A}$. Consider the left cone $\left[\bar{P}, \bar{\beta}_{i}, A_{i}\right]_{I}$ in $\mathcal{A}$, where $\bar{\beta}_{i} \rho_{P}=\beta_{i}$ for all $i \in I$. Then

$$
\alpha_{i} \bar{\beta}_{i}: \bar{P} \rightarrow A_{i} \rightarrow A
$$


are the same for all $i \in I$ (by uniqueness). By axiom $G P B_{2}$, there exists a unique morphism $\eta: \bar{P} \rightarrow P$ in $\mathcal{C}$ such that

$$
\beta_{i} \eta=\bar{\beta}_{i}: \bar{P} \rightarrow A \text { for all } i \in I .
$$

For each $i \in I$, we have

$$
\beta_{i}\left(\eta \rho_{P}\right)=\left(\beta_{i} \eta\right) \rho_{P}=\bar{\beta}_{i} \rho_{P}=\beta_{i} I_{P}
$$

Further, by axiom $G P B_{2}$, we get

$$
\eta \rho_{P}=I_{P}
$$

Now, we will show that $\rho_{P} \eta=I_{\bar{P}}$. Since $\mathcal{A}$ is a full subcategory, we can assume that

$$
\begin{gathered}
\rho_{\ddot{P}}=I_{\bar{P}}, \\
\bar{R}\left(\rho_{P}\right)=I_{\bar{P}},
\end{gathered}
$$

where $\bar{R}$ is the reflector of $\mathcal{C}$ in $\mathcal{A}$, which is a left adjoint of the inclusion functor from $\mathcal{A}$ into $\mathcal{C}$. Thus

$$
\rho_{P} \eta=\bar{R}(\eta) \rho_{\bar{P}}=\bar{R}(\eta) \bar{R}\left(\rho_{P}\right)=\bar{R}\left(\eta \rho_{P}\right)=\bar{R}\left(I_{P}\right)=I_{\bar{p}} .
$$

This shows that $\rho_{P}$ is an isomorphism and consequently, we get

$$
G P B\left[A_{i}, \alpha_{i}, A\right]_{I}=\left[\bar{P}, \bar{\beta}_{i}, A_{i}\right]_{I} \text { in } \mathcal{A} .
$$

Hence $\mathcal{A}$ is a $G P B$-category.

From Theorem 3 and Remark 3, we have

Corollary 1 : Every full reflective subcategory of a $G P B$ - category is a $G P B$ - subcategory.

From Theorem 3 and the definition of replete subcategory, we have

Corollary 2 : Every full replete reflective subcategory of a $G P B-$ category is a $G P B$ - closed subcategory.

Dually, we have

Theorem $3^{*}$ : Every full coreflective subcategory of a $G P O$ - category is also a $G P O-$ category.

Corollary $1^{*}$ : Every full replete coreflective subcategory of a $G P O-$ category is a $G P O-$ subcategory. 
Corollary $2^{*}$ : Every full replete coreflective subcategory of a $G P O-$ category is a $G P O-$ closed subcategory.

Lemma 1 : The reflection of an initial object is also an initial object.

Proof : Let $\mathcal{A}$ be a reflective subcategory of $\mathcal{C}$. Let $N \in \mathcal{C}$ be an initial object. Since for every object $A \in \mathcal{A}$, we have

$$
\operatorname{Hom}_{c}(N, A) \cong \operatorname{Hom}_{a}(\bar{N}, A) .
$$

But, $\operatorname{Hom}_{\mathcal{C}}(N, A)$ is singleton for all $A \in \mathcal{A}$. So $\operatorname{Hom}_{\mathcal{A}}(\bar{N}, A)$ is also singleton for all $A \in \mathcal{A}$. Therefore, $\bar{N}$ is an initial object in $\mathcal{A}$.

From Lcmma 1, Theorem 3 and [5, Theorem 4], we have

Theorem 4 : Every full reflective subcategory of a complete category is also right complete.

Dually, we have

Lemma $1^{*}$ : The coreflection of a terminal object is also a terminal object.

Theorem $4^{*}$ : Every full coreflective subcategory of a left complete category is also left complete.

Theorem 5 : If a category has a family of generators, then every reflective subcategory of it, also, have a family of generators.

Proof : Let $\mathcal{A}$ be a reflective subcategory of the category $\mathcal{C}$ and let $\left\{C_{i}\right\}_{i \in I}$ be a family of generators in $\mathcal{C}$.

Consider the family of objects $\left\{\bar{C}_{i}\right\}_{i \in I}$ in $\mathcal{A}$. Let $\alpha_{1}, \alpha_{2}: A \rightarrow A^{\prime}$ be an arbitrary pair of distinct morphisms in $\mathcal{A}$. By definition of family of generators, there exists a morphism $\alpha: C_{i} \rightarrow A$ for some $i \in I$ in $\mathcal{C}$ such that

$$
\begin{gathered}
\alpha_{1} \alpha \neq \alpha_{2} \alpha: C_{i} \rightarrow A^{\prime}, \\
\left(\alpha_{1} \bar{\alpha}\right) \rho_{C_{i}} \neq\left(\alpha_{i} \bar{\alpha}\right) \rho_{C_{i}}, \\
\alpha_{i} \bar{\alpha} \neq \alpha_{2} \bar{\alpha}: \bar{C}_{i} \rightarrow A^{\prime} .
\end{gathered}
$$

Hence, $\left\{\bar{C}_{i}\right\}_{i \in I}$ is a family of generators in $\mathcal{A}$. 
Dually, we have

Theorem $5^{*}$ : If a category has a family of generators, then every coreflective subcategory of it, also, have a family of cogenerators.

\section{References}

1. P. J. Freyd, Abelian categories, Harper and Row, (1964).

2. H. Herrlich and G. E. Strecker, Category Theory, Heldermann Verleg Berlin, (1979).

3. M. A. Kazim and S. M. A. Zaidi, On generalized and super pullbacks, Aligarh Bull. Math., 1, pp. 61-71, (1971).

4. M. A. Kazim and S. M. A. Zaidi, On characterization of a complete category, Tamkang J. Math., 10, pp. 205-215, (1979).

5. J. F. Kennison, A note on reflection maps Illinois J. Math., 11, pp. 404-409, (1967).

Received : March 10, 1998.

\section{Hamza A. S. Abujabal}

Department of Mathematics

Faculty of Science

King Abdul Aziz University

P. O. Box 31464

Jeddalı 21497

Saudi Arabia

and

\section{S. M. A. Zaidi}

Department of Mathematics

Aligrah Muslim University

Aligrah-202002

India 